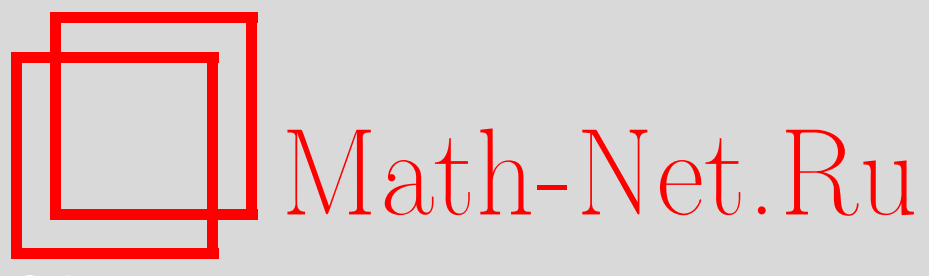

В. С. Герджиков, Е. В. Докторов, Н. П. Мацука, $N$ солитонная цепочка и обобщенная комплексная цепочка Тоды для системы Манакова, ТМФ, 2007, том 151, номер 3, 391-404

DOI: https://doi.org/10.4213/tmf6054

Использование Общероссийского математического портала Math-Net.Ru подразумевает, что вы прочитали и согласны с пользовательским соглашением http://www . mathnet.ru/rus/agreement

Параметры загрузки:

IP : 3.85 .5 .30

26 апреля 2023 г., 05:26:33

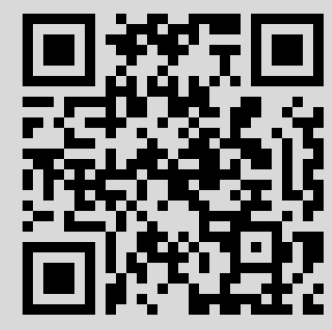




\title{
$N$-СОЛИТОННАЯ ЦЕПОЧКА И ОБОБЩЕННАЯ КОМПЛЕКСНАЯ ЦЕПОЧКА ТОДЫ ДЛЯ СИСТЕМЫ МАНАКОВА
}

\begin{abstract}
Исследуется динамическое поведение $N$-солитонной цепочки для системы Манакова и для векторного нелинейного уравнения Шредингера в адиабатическом приближении. Доказано, что в обоих случаях динамика $N$-солитонной цепочки описывается обобщенным вариантом модели комплексной цепочки Тоды. Это обстоятельство может быть использовано для предсказания асимптотических режимов $N$-солитонной цепочки при условии, что заданы начальные параметры солитонов.
\end{abstract}

Ключевые слова: комплексная цепочка Тоды, модель Манакова, адиабатическая динамика, векторная солитонная цепочка.

\section{1. ВВЕДЕНИЕ}

Получение как можно более плотной упаковки солитонов для достижения высокой скорости передачи информации представляет собой важную проблему в ориентированных на использование солитонов системах связи [1]. В результате воздействия соседних солитонов друг на друга они могут оказаться вне надлежащего временного окна при их регистрации. На примере солитонов скалярного нелинейного уравнения Шредингера (НУШ) было установлено (как численно [2], так и экспериментально [3]), что для некоторых способов выбора параметров солитонов существует возможность значительно подавить указанное солитонное взаимодействие. В адиабатическом приближении теории возмущений для солитонов НУШ проблема оптимального выбора солитонных параметров решается аналитически на основе модели комплексной цепочки Тоды (КЦТ). А именно, обобщение квазичастичного подхода Карпмана-Соловьева [4] на случай цепочки $N$ слабо взаимодействующих

${ }^{*}$ Institute for Nuclear Research and Nuclear Energy, 1784 Sofia, Bulgaria.

E-mail: gerdjikov@inrne.bas.bg

${ }^{\dagger}$ Институт физики им. В. И. Степанова, 220072, Минск, Белоруссия. E-mail: doktorov@dragon.bas-net.by

${ }^{\ddagger}$ Институт математики НАН Белоруссии, 220072, Минск, Белоруссия. E-mail: matsuka@im.bas-net.by 
солитонов НУШ приводит к $4 N$-мерной динамической системе эволюционных уравнений для солитонных параметров [5]. При некоторых дополнительных предположениях эта динамическая система сводится к вполне интегрируемой модели КЦТ с $N$ узлами [6]. Этот подход был распространен на некоторые другие нелинейные эволюционные уравнения, в числе которых модифицированное НУШ [7], массивная модель Тирринга [8], система Абловица-Ладика [9] и другие, что дает веские основания считать КЦТ универсальной моделью для солитонных конфигураций типа цепочек. Возможность вывести модель КЦТ для динамики солитонных цепочек с помощью метода Лагранжа представляет собой дополнительный аргумент в пользу такой универсальности [10].

Новые характерные черты поведения солитонов внутри цепочек появляются в случае векторных солитонов. Действительно, оптические волокна являются двулучепреломляющими, и импульсы распространяются вдоль волокна с различными скоростями по двум ортогональным направлениям поляризации. Усредняя флуктуации двулучепреломления, вызванные случайными изгибами и скручиванием волокна, а также воздействием окружающей среды, и пренебрегая дисперсией поляризационных мод, мы приходим к системе Манакова для моделирования динамики векторных солитонов [11]

$$
\begin{aligned}
i u_{t}+\frac{1}{2} u_{x x}+\left(|u|^{2}+|v|^{2}\right) u & =0 \\
i v_{t}+\frac{1}{2} v_{x x}+\left(|u|^{2}+|v|^{2}\right) v & =0
\end{aligned}
$$

где $u$ и $v$ - поляризационные составляющие медленно меняющейся огибающей, а также приняты математически более удобные обозначения для координат: $t$ и $x$ (вместо принятых в физике $z$ и $t$ ). Солитонное решение системы (1) хорошо известно:

$$
\left(\begin{array}{l}
u \\
v
\end{array}\right)=2 \nu\left(\begin{array}{l}
e^{i \chi^{(1)}} \cos \theta \\
e^{i \chi^{(2)}} \sin \theta
\end{array}\right) e^{2 i \mu(x-\xi)} \operatorname{sch}(2 \nu(x-\xi)),
$$

где

$$
\xi=2 \mu t+\xi^{(0)}, \quad \chi^{(j)}=2 \mu \xi-2\left(\mu^{2}-\nu^{2}\right) t+\delta^{(j)} .
$$

Вещественные параметры $\mu$ и $\nu$ определяют соответственно скорость и амплитуду солитона, через $\xi^{(0)}$ и $\delta^{(j)}$ обозначены начальное положение солитона и его фазы, $\theta-$ угол поляризации.

Типичный случай $N$ солитонов, движущихся с попарно различными скоростями (и поэтому являющихся асимптотически свободными при $|x| \rightarrow \pm \infty$ ), допускает полностью аналитическое описание в рамках метода обратной спектральной задачи [11]-[13]. Нам, однако, предстоит иметь дело с конфигурацией типа цепочки, состоящей из упорядоченной последовательности $N$ слабо взаимодействующих солитонов с равными или почти равными амплитудами и скоростями, расстояния между которыми практически одинаковы. Мы намерены вывести некоторую модель КЦТ для векторных солитонов и исследовать различные асимптотические режимы эволюции цепочек. 
Случай двух взаимодействующих солитонов Манакова был рассмотрен Янгом [14]. Он исследовал зависимость радиуса взаимодействия солитонов от состояния их поляризации друг относительно друга. В этой связи следует подчеркнуть, что поскольку динамическая система Карпмана-Соловьева является нелинейной, то для нее не выполняется принцип суперпозиции. Поэтому поведение цепочки с $N \geqslant 3$ солитонами невозможно описать на основе двухсолитонного взаимодействия.

\section{2. ВОЗМУЩЕННАЯ СИСТЕМА МАНАКОВА: ПОДХОД КАРПМАНА-СОЛОВЬЕВА}

Предположим, что $N$-солитонное решение системы Манакова (1) для солитонной цепочки определяется последовательностью начальных односолитонных импульсов с перекрывающимися хвостами

$$
\left(\begin{array}{l}
u \\
v
\end{array}\right)(x, 0)=\sum_{k=1}^{N} 2 \nu_{k}\left(\begin{array}{l}
e^{i\left(2 \mu_{k} \xi_{k}^{(0)}+\delta_{k}^{(1)}\right)} \cos \theta_{k} \\
e^{i\left(2 \mu_{k} \xi_{k}^{(0)}+\delta_{k}^{(2)}\right)} \sin \theta_{k}
\end{array}\right) e^{2 i \mu_{k}\left(x-\xi_{k}^{(0)}\right)} \operatorname{sch}\left(2 \nu_{k}\left(x-\xi_{k}^{(0)}\right)\right) .
$$

Каждый солитон характеризуется представленным выше множеством параметров, которое мы снабдим индексом $k$ (номером солитона). Малость величины перекрытия соседних солитонов определяется параметром

$$
\epsilon=\left|\int_{-\infty}^{\infty} d x u_{k}^{\dagger}(x) u_{n}(x)\right|=\left|\int_{-\infty}^{\infty} d x \frac{4 \nu_{k} \nu_{n} e^{i\left(\phi_{n}-\phi_{k}\right)}}{\operatorname{ch} z_{k} \operatorname{ch} z_{n}}\right| \simeq 8 \nu_{0} \Delta_{k n} e^{-\Delta_{k n}},
$$

где $n=k \pm 1, \nu_{0}$ - среднее значение амплитуд солитонов, $\nu_{0}=(1 / N) \sum_{k} \nu_{k}$ и $\Delta_{k, n}=2 \nu_{0}\left|\xi_{k}^{(0)}-\xi_{n}^{(0)}\right|$. При выводе формулы (3) была использована асимптотическая формула для функции $K(0, \Delta)$ при больших $\Delta$ (см. приложение). Ограничимся учетом взаимодействия между ближайшими соседями и будем считать, что сила взаимодействия между соседними солитонами по порядку величины равна величине их перекрытия.

Следуя подходу Карпмана-Соловьева [4], можно выписать адиабатическое условие для солитонного взаимодействия в виде

$$
\begin{gathered}
\left|\nu_{k}-\nu_{k \pm 1}\right| \ll \nu_{0}, \quad\left|\mu_{k}-\mu_{k \pm 1}\right| \ll \nu_{0}, \\
\nu_{0}\left|\xi_{k}-\xi_{k \pm 1}\right| \gg 1, \quad\left|\nu_{k}-\nu_{0}\right|\left|\xi_{k}-\xi_{k \pm 1}\right| \ll 1,
\end{gathered}
$$

обеспечивающем малость величины $\epsilon$ (3). В неравенствах (4) находит свое математическое выражение то обстоятельство, что мы рассматриваем эволюцию цепочки слабо перекрывающихся солитонов Манакова с (почти) равными амплитудами и малыми относительными скоростями.

Подставляя в систему (1) решение в виде суммы

$$
\left(\begin{array}{l}
u \\
v
\end{array}\right)=\sum_{k=1}^{N}\left(\begin{array}{l}
u_{k} \\
v_{k}
\end{array}\right)
$$


мы видим, что $k$-й солитон удовлетворяет возмущенной системе Манакова

$$
\begin{aligned}
& i u_{k t}+\frac{1}{2} u_{k x x}+\left(\left|u_{k}\right|^{2}+\left|v_{k}\right|^{2}\right) u_{k}=i r_{k}, \\
& i v_{k t}+\frac{1}{2} v_{k x x}+\left(\left|u_{k}\right|^{2}+\left|v_{k}\right|^{2}\right) v_{k}=i s_{k},
\end{aligned}
$$

где возмущения

$$
\begin{gathered}
r_{k}=i\left(u_{k-1}+u_{k+1}\right)\left(2\left|u_{k}\right|^{2}+\left|v_{k}\right|^{2}\right)+i\left(\bar{u}_{k-1}+\bar{u}_{k+1}\right) u_{k}^{2}+ \\
+i u_{k}\left(v_{k-1} \bar{v}_{k}+\bar{v}_{k-1} v_{k}+v_{k} \bar{v}_{k+1}+\bar{v}_{k} v_{k+1}\right) \\
s_{k}=i\left(v_{k-1}+v_{k+1}\right)\left(2\left|v_{k}\right|^{2}+\left|u_{k}\right|^{2}\right)+i\left(\bar{v}_{k-1}+\bar{v}_{k+1}\right) v_{k}^{2}+ \\
+i v_{k}\left(u_{k-1} \bar{u}_{k}+\bar{u}_{k-1} u_{k}+u_{k} \bar{u}_{k+1}+\bar{u}_{k} u_{k+1}\right)
\end{gathered}
$$

определяются взаимодействием левого и правого соседних солитонов с $k$-м солитоном. Вызванная возмущениями эволюция параметров солитонов Манакова в адиабатическом приближении была получена ранее [15] и в нашем случае записывается в виде

где

$$
\begin{aligned}
\frac{d \mu_{k}}{d t} & =\frac{1}{2} \int_{-\infty}^{\infty} d z_{k} \operatorname{sch} z_{k} \operatorname{th} z_{k} \operatorname{Im} R_{k}^{(+)}, \\
\frac{d \nu_{k}}{d t} & =\frac{1}{2} \int_{-\infty}^{\infty} d z_{k} \operatorname{sch} z_{k} \operatorname{Re} R_{k}^{(+)}, \\
\frac{d \theta_{k}}{d t} & =-\frac{1}{4 \nu_{k}} \int_{-\infty}^{\infty} d z_{k} \operatorname{sch} z_{k} \operatorname{Re} R_{k}^{(-)}=O(\epsilon), \\
\frac{d \xi_{k}}{d t} & =2 \mu_{k}+O(\epsilon), \quad \frac{d \chi_{k}^{(j)}}{d t}=2\left(\mu_{k}^{2}+\nu_{k}^{2}\right)+O(\epsilon),
\end{aligned}
$$

$$
\begin{gathered}
z_{k}=2 \nu_{k}\left(x-\xi_{k}\right), \quad R_{k}^{(+)}=R_{k} \cos \theta_{k}+S_{k} \sin \theta_{k}, \quad R_{k}^{(-)}=R_{k} \sin \theta_{k}-S_{k} \cos \theta_{k}, \\
R_{k}=r_{k} \exp \left(-i \frac{\mu_{k}}{\nu_{k}} z_{k}-i \chi_{k}^{(1)}\right), \quad S_{k}=s_{k} \exp \left(-i \frac{\mu_{k}}{\nu_{k}} z_{k}-i \chi_{k}^{(2)}\right) .
\end{gathered}
$$

Нет необходимости конкретизировать точный вид выражений $O(\epsilon)$, поскольку далее будет показано, что их вклад в окончательные выражения имеет более высокий порядок по $\epsilon$.

Подставляя теперь солитонное решение (2), снабженное индексом $k$, в формулы для возмущений (6) и производя вычисления в соответствии с формулами (7), получаем эволюционные уравнения для скорости и амплитуды возмущенного $k$-го солитона:

$$
\frac{d \mu_{k}}{d t}=-16 \nu_{0}^{3} \sum_{n=k \pm 1} s_{k n} e^{-\Delta_{k n}} P_{k n}^{(1)}, \quad \frac{d \nu_{k}}{d t}=16 \nu_{0}^{3} \sum_{n=k \pm 1} s_{k n} e^{-\Delta_{k n}} P_{k n}^{(2)},
$$

где $s_{k, k+1}=-1, s_{k, k-1}=1$,

$$
\begin{gathered}
P_{k n}^{(1)}=\cos \theta_{k} \cos \theta_{n} \cos \alpha_{k n}^{(1)}+\sin \theta_{k} \sin \theta_{n} \cos \alpha_{k n}^{(2)}, \\
P_{k n}^{(2)}=\cos \theta_{k} \cos \theta_{n} \sin \alpha_{k n}^{(1)}+\sin \theta_{k} \sin \theta_{n} \sin \alpha_{k n}^{(2)}, \\
\Delta_{k n}=2 \nu_{0} s_{k n}\left(\xi_{k}-\xi_{n}\right), \quad \alpha_{k n}^{(j)}=\chi_{k}^{(j)}-\chi_{n}^{(j)}-2 i \mu_{0}\left(\xi_{k}-\xi_{n}\right),
\end{gathered}
$$


а эволюция других параметров определяется формулами (7). Заметим, что нетрудно вывести и явные формулы для эволюции оставшихся солитонных параметров. Например, угол поляризации удовлетворяет уравнению

$$
\begin{gathered}
\frac{d \theta_{k}}{d t}=-8 \nu_{0}^{2} \sum_{n=k \pm 1} s_{k n} e^{-\Delta_{k n}} P_{k n}^{(3)}, \\
P_{k n}^{(3)}=\sin \theta_{k} \cos \theta_{n} \sin \alpha_{k n}^{(1)}-\cos \theta_{k} \sin \theta_{n} \sin \alpha_{k n}^{(2)} .
\end{gathered}
$$

Однако, как уже утверждалось выше, их вклад пренебрежимо мал по сравнению с главным порядком по $\epsilon \sim e^{-\Delta_{k n}}$.

\section{3. ВЫВОД МОДЕЛИ КЦТ}

Введем величину $\lambda_{k}=\mu_{k}+i \nu_{k}-\left(\mu_{0}+i \nu_{0}\right)$, где $\mu_{0}$ и $\nu_{0}$ суть средние значения. Тогда из уравнений (8) следует, что

$$
\frac{d \lambda_{k}}{d t}=16 \nu_{0}^{3}\left(e^{2 i\left(\mu_{0}+i \nu_{0}\right)\left(\xi_{k+1}-\xi_{k}\right)}\left\langle\vec{n}_{k+1}, \vec{n}_{k}\right\rangle-e^{2 i\left(\mu_{0}+i \nu_{0}\right)\left(\xi_{k}-\xi_{k-1}\right)}\left\langle\vec{n}_{k}, \vec{n}_{k-1}\right\rangle\right) .
$$

Здесь введен двухкомпонентный вектор $\left|\vec{n}_{k}\right\rangle$ и не зависящая от поляризации фаза $\tilde{\chi}_{k}$,

$$
\left|\vec{n}_{k}\right\rangle=\left(\begin{array}{c}
e^{i \chi^{(1)}} \cos \theta_{k} \\
e^{i \chi^{(2)}} \sin \theta_{k}
\end{array}\right), \quad\left\langle\vec{n}_{k}, \vec{n}_{k \pm 1}\right\rangle=e^{-i\left(\tilde{\chi}_{k}-\tilde{\chi}_{k \pm 1}\right)}\left\langle\vec{n}_{k}^{0}, \vec{n}_{k \pm 1}^{0}\right\rangle, \quad \chi_{k}^{(j)}=\tilde{\chi}_{k}+\delta_{k}^{(j)},
$$

где вектор поляризации $\left|\vec{n}_{k}^{0}\right\rangle$ дается формулами

$$
\begin{gathered}
\left|\vec{n}_{k}^{0}\right\rangle=\left(\begin{array}{c}
e^{i \delta^{(1)}} \cos \theta \\
e^{i \delta^{(2)}} \sin \theta
\end{array}\right), \quad\left\langle\vec{n}_{k}^{0}, \vec{n}_{k \pm 1}^{0}\right\rangle \equiv\left(\vec{n}_{k}^{0 \dagger}, \vec{n}_{k \pm 1}^{0}\right), \\
\left\langle\vec{n}_{k}^{0}, \vec{n}_{k \pm 1}^{0}\right\rangle=e^{-i\left(\delta_{k}^{(1)}-\delta_{k \pm 1}^{(1)}\right)} \cos \theta_{k} \cos \theta_{k \pm 1}+e^{-i\left(\delta_{k}^{(2)}-\delta_{k \pm 1}^{(2)}\right)} \sin \theta_{k} \sin \theta_{k \pm 1} .
\end{gathered}
$$

Кроме того,

$$
\frac{d \tilde{\chi}_{k}}{d t}=2\left(\mu_{k}^{2}+\nu_{k}^{2}\right)+O(\epsilon), \quad \frac{d}{d t}\left\langle\vec{n}_{k}^{0}, \vec{n}_{k-1}^{0}\right\rangle=O(\epsilon) .
$$

Введем теперь величины

$$
\begin{aligned}
& E_{k+1, k}=4 \nu_{0}^{2} \exp \left[2 i\left(\mu_{0}+i \nu_{0}\right)\left(\xi_{k+1}-\xi_{k}\right)-i\left(\tilde{\chi}_{k+1}-\tilde{\chi}_{k}\right)\right], \\
& E_{k, k-1}=4 \nu_{0}^{2} \exp \left[2 i\left(\mu_{0}+i \nu_{0}\right)\left(\xi_{k}-\xi_{k-1}\right)-i\left(\tilde{\chi}_{k}-\tilde{\chi}_{k-1}\right)\right],
\end{aligned}
$$

которые удовлетворяют уравнениям

$$
\frac{d E_{k+1, k}}{d t}=-4 \nu_{0}\left(\lambda_{k+1}-\lambda_{k}\right) E_{k+1, k}, \quad \frac{d E_{k, k-1}}{d t}=-4 \nu_{0}\left(\lambda_{k}-\lambda_{k-1}\right) E_{k, k-1} .
$$

Тогда

$$
\frac{d \lambda_{k}}{d t}=4 \nu_{0}\left[E_{k+1, k}\left\langle\vec{n}_{k+1}^{0}, \vec{n}_{k}^{0}\right\rangle-E_{k, k-1}\left\langle\vec{n}_{k}^{0}, \vec{n}_{k-1}^{0}\right\rangle\right] .
$$

Видно, что для попарно ортогонально поляризованных решений (все произведения $\left\langle\vec{n}_{k}^{0}, \vec{n}_{k-1}^{0}\right\rangle$ равны нулю) как скорость, так и амплитуда $k$-го солитона остаются постоянными в главном порядке по $\epsilon$, т.е. солитоны не взаимодействуют. 
Пусть $E_{k, k-1}=-e^{q_{k}-q_{k-1}}$ и аналогичное равенство имеет место для $E_{k+1, k}$. Тогда $q_{k t}=-4 \nu_{0} \lambda_{k}$ и

$$
\frac{d^{2} q_{k}}{d t^{2}}=16 \nu_{0}^{2}\left[\left\langle\vec{n}_{k+1}^{0}, \vec{n}_{k}^{0}\right\rangle e^{q_{k+1}-q_{k}}-\left\langle\vec{n}_{k}^{0}, \vec{n}_{k-1}^{0}\right\rangle e^{q_{k}-q_{k-1}}\right]
$$

где

$$
q_{k}=2 i\left(\mu_{0}+i \nu_{0}\right)\left[\xi_{k}-\left(\mu_{0}+i \nu_{0}\right) t\right]-i \tilde{\chi}_{k}+k \ln 4 \nu_{0}^{2}+i k \pi .
$$

Определим теперь величины $Q_{k}$ :

$$
Q_{1}=q_{1}, \quad Q_{k}=q_{k}+\sum_{s=2}^{k} \ln \left\langle\vec{n}_{s}^{0}, \vec{n}_{s-1}^{0}\right\rangle,
$$

и нормированное время $\tau=4 \nu_{0} t$. Тогда уравнение (9) в точности приобретает вид вполне интегрируемой модели КЦТ с $N$ узлами:

$$
\frac{d^{2} Q_{k}}{d \tau^{2}}=e^{Q_{k+1}-Q_{k}}-e^{Q_{k}-Q_{k-1}} .
$$

Решающим является то обстоятельство, что мы получили комплексную модель Тоды. Хорошо известно [16], что вещественная цепочка Тоды (ВЦТ) с $N$ узлами допускает $L-A$-пару и имеет $N$ попарно различных вещественных интегралов движения, находящихся в инволюции. Поскольку эти интегралы определяют асимптотические скорости “частиц ВЦТ”, мы приходим к заключению, что все эти частицы имеют попарно различные скорости и эволюционируют к асимптотически свободному режиму.

Аналогично, модель КЦТ имеет $N$ комплексных интегралов движения и асимптотические скорости даются вещественными частями этих интегралов. Следовательно, мы можем иметь, вообще говоря, различные комплексные интегралы движения с равными вещественными частями. Такое положение отвечает режиму связанных состояний, когда вещественные части всех интегралов одинаковы, или промежуточному режиму, когда равны вещественные части лишь некоторых из интегралов движения (см. [17]).

Заметим, что если $\left\langle\vec{n}_{k}^{\dagger}, \vec{n}_{k \pm 1}\right\rangle=0$ для некоторого фиксированного значения $k$, то, очевидно, модель КЦТ (10) расщепляется на две отдельные системы КЦТ: первая для солитонов с индексами $1 \leqslant s \leqslant k$, вторая - с индексами $k+1 \leqslant s \leqslant N$. Далее мы не будем иметь дело с этим вырожденным случаем.

\section{4. ТРЕХСОЛИТОННЫЕ ВЗАИМОДЕЙСТВИЯ}

Для иллюстрации вышеизложенного подхода рассмотрим распространение цепочки солитонов Манакова с $N=3$, опуская некоторые детали вычислений, поскольку они представляют собой очевидное обобщение аналогичных результатов для скалярных солитонов НУШ [18]. Возьмем

$$
Q_{1}=q_{1}-\ln \left\langle\vec{n}_{2}^{0}, \vec{n}_{1}^{0}\right\rangle, \quad Q_{2}=q_{2}, \quad Q_{3}=q_{3}+\ln \left\langle\vec{n}_{3}^{0}, \vec{n}_{2}^{0}\right\rangle .
$$


Матрица $L$ соответствующей спектральной задачи имеет вид

$$
L=\left(\begin{array}{ccc}
b_{1} & a_{1} & 0 \\
a_{1} & b_{2} & a_{2} \\
0 & a_{2} & b_{3}
\end{array}\right)
$$

где

$$
a_{k}=\frac{1}{2} e^{\left(Q_{k+1}-Q_{k}\right) / 2}, \quad b_{k}=-\frac{1}{2} \frac{d Q_{k}}{d \tau}, \quad \sum_{k=1}^{3} b_{k}=0 .
$$

Интегралы движения $\zeta_{k}$ являются собственными значениями матрицы $L$. Таким образом, для исследования вещественных частей этих интегралов можно рассмотреть начальные параметры солитонов. Для простоты возьмем начальные скорости равными нулю $\left(\mu_{j 0}=0\right)$. Тогда $q_{k}-q_{k-1}=-2 \nu_{0} r_{0}+\ln 4 \nu_{0}^{2}+i \pi$, где $r_{0}$ - начальное расстояние между солитонами, поэтому

$$
\begin{aligned}
a_{1} & =i \nu_{0} \sqrt{\left\langle\vec{n}_{2}^{0}, \vec{n}_{1}^{0}\right\rangle} e^{-\nu_{0} r_{0}}, & a_{2}=i \nu_{0} \sqrt{\left\langle\vec{n}_{3}^{0}, \vec{n}_{2}^{0}\right\rangle} e^{-\nu_{0} r_{0}}, \\
b_{1}=\frac{i}{2}\left(\nu_{1}-\nu_{0}\right) \equiv \frac{i}{2} \Delta \nu_{1}, & b_{2}=\frac{i}{2} \Delta \nu_{2}, & b_{3}=\frac{i}{2} \Delta \nu_{3} .
\end{aligned}
$$

Собственные значения матрицы $L$ являются решениями алгебраического уравнения

$$
\zeta^{3}+p \zeta+q=0
$$

где

$$
\begin{gathered}
p=\nu_{0}^{2} e^{-2 \nu_{0} r_{0}}\left(\left\langle\vec{n}_{2}^{0}, \vec{n}_{1}^{0}\right\rangle+\left\langle\vec{n}_{3}^{0}, \vec{n}_{2}^{0}\right\rangle\right)-\frac{1}{4}\left(\Delta \nu_{1} \Delta \nu_{2}+\Delta \nu_{2} \Delta \nu_{3}+\Delta \nu_{3} \Delta \nu_{1}\right), \\
q=\frac{i}{8} \Delta \nu_{1} \Delta \nu_{2} \Delta \nu_{3}-\frac{i}{2} \nu_{0}^{2} e^{-2 \nu_{0} r_{0}}\left(\left\langle\vec{n}_{2}^{0}, \vec{n}_{1}^{0}\right\rangle \Delta \nu_{3}+\left\langle\vec{n}_{3}^{0}, \vec{n}_{2}^{0}\right\rangle \Delta \nu_{1}\right) .
\end{gathered}
$$

Простой анализ уравнения третьей степени (11) с дискриминантом $Q=(q / 2)^{2}+$ $(p / 3)^{3}$ показывает, что существуют две возможности для эволюции начальных солитонных параметров в режим связанного состояния: 1) $p>0, q=0$; $) p=\bar{p}$, $q=-\bar{q}, Q>0$.

Рассмотрим случай 1 . Для определенности будем рассматривать следующую конфигурацию величин $\Delta \nu_{k}: \Delta \nu_{1}=\beta, \Delta \nu_{2}=0, \Delta \nu_{3}=-\beta$. Чтобы величина $p$ была вещественной, нужно обратить в нуль величину $\operatorname{Im}\left(\left\langle\vec{n}_{2}^{0}, \vec{n}_{1}^{0}\right\rangle+\left\langle\vec{n}_{3}^{0}, \vec{n}_{2}^{0}\right\rangle\right)$. Одна из возможностей состоит в том, чтобы положить

$$
\delta_{1}^{(1)}=\delta_{2}^{(1)}=\delta_{3}^{(1)}, \quad \delta_{1}^{(2)}=\delta_{2}^{(2)}=\delta_{3}^{(2)}
$$

для любых $\theta_{1}$ и $\theta_{2}$, без всякой корреляции между параметрами $\delta_{k}^{(1)}$ и $\delta_{k}^{(2)}$. Тогда

$$
p=\nu_{0}^{2} e^{-2 \nu_{0} r_{0}}\left(\cos \theta_{21}+\cos \theta_{32}\right)+\frac{1}{4} \beta^{2}, \quad q=\frac{i}{2} \nu_{0}^{2} \beta e^{-2 \nu_{0} r_{0}}\left(\cos \theta_{21}-\cos \theta_{32}\right),
$$

где $\theta_{k n} \equiv \theta_{k}-\theta_{n}$. Имеем $q=0$, если $\theta_{21}=\theta_{32}$, что дает $\theta_{2}=\left(\theta_{1}+\theta_{3}\right) / 2$. Следовательно, $\theta_{21}=\theta_{32}=\theta_{31} / 2$ и

$$
p=2 \nu_{0}^{2} e^{-2 \nu_{0} r_{0}} \cos \left(\frac{1}{2} \theta_{31}\right)+\frac{1}{4} \beta^{2} .
$$


В результате, $p>0$ в том случае, если

$$
-\frac{\pi}{2}<\frac{\left|\theta_{1}-\theta_{3}\right|}{2}<\frac{\pi}{2}
$$

для любого $\beta$ (напомним, что величина $\beta$ должна быть мала в силу (4)) или если

$$
\frac{\pi}{2}<\frac{\left|\theta_{1}-\theta_{3}\right|}{2}<\frac{3 \pi}{2}, \quad \beta>2 \sqrt{2} \nu_{0} e^{-\nu_{0} r_{0}} .
$$

Обратим внимание на появление порогового условия для $\beta$ [18].

Проиллюстрируем случай 2 примером другой конфигурации величин $\Delta \nu_{k}$ : $\Delta \nu_{1}=\Delta \nu_{3}=\gamma, \Delta \nu_{2}=-2 \gamma$. В этом случае

$$
\begin{gathered}
p=\nu_{0}^{2} e^{-2 \nu_{0} r_{0}}\left(\cos \theta_{21}+\cos \theta_{32}\right)+\frac{3}{4} \gamma^{2} \\
q=-\frac{i}{4} \gamma\left[2 \nu_{0}^{2} e^{-2 \nu_{0} r_{0}}\left(\cos \theta_{21}+\cos \theta_{32}\right)+\gamma^{2}\right] \\
Q=\left(\frac{\nu_{0}}{2} e^{-\nu_{0} r_{0}}\right)^{4} \frac{\gamma^{2}}{3}\left(\cos \theta_{21}+\cos \theta_{32}\right)^{2}\left[1+\frac{16}{9}\left(\frac{\nu_{0}}{\gamma} e^{-\nu_{0} r_{0}}\right)^{2}\left(\cos \theta_{21}+\cos \theta_{32}\right)\right] .
\end{gathered}
$$

Мы получаем, что $Q>0$, если выражение в квадратных скобках положительно. Это дает пороговое условие для параметра $\gamma$ :

$$
\gamma>\frac{4 \sqrt{2}}{3} \nu_{0} e^{-\nu_{0} r_{0}} .
$$

Симметричные решения КЦТ при $N=3$ в случае $p>0$ и $q=0$ могут быть явно выписаны в виде [18]

$$
Q_{1}(\tau)=\ln \frac{\operatorname{ch}\left(2 \zeta_{1} \tau+\sigma\right)+1}{4 \zeta_{1}^{2}}, \quad Q_{2}=0, \quad Q_{3}=-Q_{1},
$$

где $\zeta_{1}=\sqrt{-p}, \sigma=\rho_{+}$при $\cos \left(\theta_{31} / 2\right)>0$ и $\sigma=\rho_{-}+i \pi$ при $\cos \left(\theta_{31} / 2\right)<0($ см. ниже). Следуя [18], можно вывести формулы для траекторий солитонов:

$$
\begin{aligned}
& \xi_{1}(\tau)=-\frac{1}{2 \nu_{0}}\left[\operatorname{Re} Q_{1}(\tau)+\ln \left|\cos \frac{\theta_{3}-\theta_{1}}{2}\right|-\ln \left(4 \nu_{0}^{2}\right)\right], \\
& \xi_{2}(\tau)=\frac{1}{\nu_{0}} \ln \left(4 \nu_{0}^{2}\right), \\
& \xi_{3}(\tau)=\frac{1}{2 \nu_{0}}\left[\operatorname{Re} Q_{1}(\tau)+\ln \left|\cos \frac{\theta_{3}-\theta_{1}}{2}\right|+3 \ln \left(4 \nu_{0}^{2}\right)\right],
\end{aligned}
$$

где

$$
\begin{gathered}
\operatorname{Re} Q_{1}(\tau)=\ln [\operatorname{ch}(\operatorname{Re} \sigma)+\cos (2 \sqrt{p} \tau+\operatorname{Im} \sigma)]-\ln (4 p), \\
\operatorname{ch} \rho_{+}=\frac{C_{+}^{2}+1}{C_{+}^{2}-1}, \quad \operatorname{ch} \rho_{-}=\frac{1+C_{-}^{2}}{1-C_{-}^{2}}, \\
C_{ \pm}=\left(1 \pm 8 \frac{\nu_{0}^{2}}{\beta^{2}} e^{-2 \nu_{0} r_{0}}\left|\cos \frac{\theta_{3}-\theta_{1}}{2}\right|\right)^{1 / 2}
\end{gathered}
$$


и $\beta$ удовлетворяет пороговому условию (13). Таким образом, расстояние между солитонами

$$
r(\tau)=\frac{1}{2}\left(\xi_{3}(\tau)-\xi_{1}(\tau)\right)=\frac{1}{2 \nu_{0}}\left[\operatorname{Re} Q_{1}(\tau)+\ln \left|\cos \frac{\theta_{3}-\theta_{1}}{2}\right|+2 \ln \left(4 \nu_{0}^{2}\right)\right]
$$

колеблется между максимальным и минимальным значениями с периодом $T=$ $2 \pi /\left(\beta C_{ \pm}\right)$, причем

$$
r_{\max }=r_{0}, \quad r_{\min }=r_{0}-\frac{1}{\nu_{0}} \ln C_{+}
$$

в случае $\cos \left(\theta_{31} / 2\right)>0$ и

$$
r_{\max }=r_{0}-\frac{1}{\nu_{0}} \ln C_{-}, \quad r_{\min }=r_{0}
$$

в случае $\cos \left(\theta_{31} / 2\right)<0$ (напомним, что $\left.C_{-}<1\right)$.
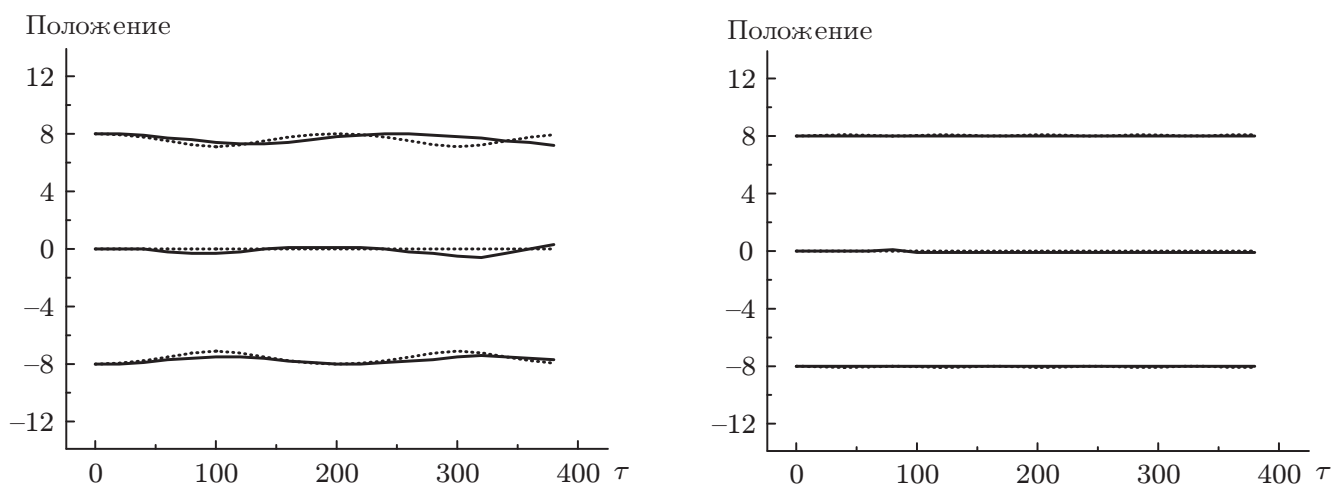

Квазиэквидистантное распространение трехсолитонной цепочки: аналитически предсказанные траектории $\xi_{j}, j=1,2,3$ (пунктирные линии) и полученные численно максимумы интенсивности солитонов $\left(\left|u_{j}\right|^{2}+\left|v_{j}\right|^{2}\right)^{1 / 2}$ (сплошные линии); слева: $r_{0}=8, \nu_{0}=0.5, \theta_{1}=0, \theta_{2}=\pi / 3, \theta_{3}=2 \pi / 3, \beta=0.02$, пороговое условие для $\beta$ отсутствует; справа: $r_{0}=8, \nu_{0}=0.5, \theta_{1}=0$, $\theta_{2}=2 \pi / 3, \theta_{3}=4 \pi / 3, \beta=0.08$, для $\beta$ выполнено пороговое условие $(13)$. Вычисленные и предсказанные траектории во втором случае практически совпадают.

Сравним теперь наши теоретические предсказания относительно режимов связанных состояний с результатами численного моделирования для случая $r_{0}=8$ и $\nu_{0}=0.5$. Для углов поляризации $\theta_{1}=0, \theta_{2}=\pi / 3$ и $\theta_{3}=2 \pi / 3$ имеем $\cos \left(\theta_{31} / 2\right)>0$. Следовательно, пороговое ограничение на значение $\beta$ отсутствует, и можно взять $\beta=0.02$. Для другого выбора углов $\left(\theta_{1}=0, \theta_{2}=2 \pi / 3\right.$ и $\left.\theta_{3}=4 \pi / 3\right)$ имеем $\cos \left(\theta_{31} / 2\right)<0$. Поэтому для выполнения порогового условия (13) возьмем $\beta=0.08$. Результаты сравнения траекторий солитонов (14) с полученными численно максимумами интенсивности солитонов $\left(\left|u_{j}\right|^{2}+\left|v_{j}\right|^{2}\right)^{1 / 2}$ представлены на рисунке. Видно, что модель КЦТ правильно предсказывает квазиэквидистантные режимы динамики цепочки для больших временных интервалов, особенно хорошее соответствие наблюдается при втором способе выбора углов поляризации. 


\section{5. МНОГОКОМПОНЕНТНАЯ СИСТЕМА НУШ: ВАРИАЦИОННЫЙ ПОДХОД}

В этом разделе мы рассмотрим $N$-солитонные взаимодействия в более общей многокомпонентной системе НУШ, используя вариационный подход [19].

Применим этот подход к векторному НУШ

$$
i \frac{d \vec{u}}{d t}+\frac{1}{2} \frac{d^{2} \vec{u}}{d x^{2}}+\langle\vec{u}, \vec{u}\rangle \vec{u}(x, t)=0,
$$

где $\vec{u}-n$-компонентная векторнозначная функция и $\langle\vec{u}, \vec{w}\rangle \equiv \sum_{s=1}^{n} \vec{u}_{s}^{*} \vec{w}-$ скалярное произведение. При $n=2$ уравнение (16) превращается в систему Манакова (1).

Уравнение (16) допускает как гамильтонову, так и лагранжеву формулировку. Действительно, вводя скобки Пуассона

$$
\left\{\vec{u}_{j}(x, t), \vec{u}_{k}^{*}(y, t)\right\}=\delta_{j k} \delta(x-y)
$$

и гамильтониан

$$
H=\int_{-\infty}^{\infty} d x\left[\frac{1}{2}\left\langle\vec{u}_{x}, \vec{u}_{x}\right\rangle-\frac{1}{2}\langle\vec{u}, \vec{u}\rangle^{2}\right],
$$

легко находим, что уравнения движения Гамильтона

$$
i \vec{u}_{j, t}=\left\{H, \vec{u}_{j}\right\} \equiv \frac{\partial H}{\partial \vec{u}_{j}^{*}}
$$

совпадают с векторным НУШ (16).

Лагранжева формулировка состоит в следующем: введем лагранжиан

$$
\mathcal{L}=\int_{-\infty}^{\infty} d t \frac{i}{2}\left[\left\langle\vec{u}, \vec{u}_{t}\right\rangle-\left\langle\vec{u}_{t}, \vec{u}\right\rangle\right]-H .
$$

Тогда уравнения движения Лагранжа

$$
\frac{d}{d t} \frac{\delta \mathcal{L}}{\delta \vec{u}_{t}^{\dagger}}-\frac{\delta \mathcal{L}}{\delta \vec{u}^{\dagger}}=0
$$

совпадают с уравнением (16).

Векторная система НУШ может быть решена посредством применения метода обратной задачи к обобщенной системе Захарова-Шабата (см. [11], [13]). Здесь мы исследуем поведение системы на больших временах при начальном условии типа цепочки

$$
\vec{u}(x)=\left.\sum_{k=1}^{N} \vec{u}_{k}(x, t)\right|_{t=0},
$$

где $\vec{u}_{k}(x, t)$ - односолитонное решение:

$$
\begin{gathered}
\vec{u}_{k}(x, t)=\frac{2 \nu_{k} e^{i \phi_{k}}}{\operatorname{ch} z_{k}} \vec{n}_{k}, \quad \vec{n}_{k}=\left(\begin{array}{c}
\kappa_{k}^{(1)} e^{i \gamma_{k}^{(1)}} \\
\vdots \\
\kappa_{k}^{(n)} e^{i \gamma_{k}^{(n)}}
\end{array}\right), \\
z_{k}=2 \nu_{k}\left(x-\xi_{k}(t)\right), \quad \xi_{k}(t)=2 \mu_{k} t+\xi_{k}^{(0)}, \\
\phi_{k}=\frac{\mu_{k}}{\nu_{k}} z_{k}+\delta_{k}(t), \quad \delta_{k}(t)=2\left(\mu_{k}^{2}+\nu_{k}^{2}\right) t+\delta_{k}^{(0)} .
\end{gathered}
$$


Введенные выше векторы поляризации $\vec{n}_{k}$ нормированы условием $\left\langle\vec{n}_{k}, \vec{n}_{k}\right\rangle \equiv$ $\sum_{s=1}^{n} \kappa_{k}^{(s) 2}=1$. Фазы $\phi_{k}$ однозначно фиксированы требованием $\sum_{s=1}^{n} \gamma_{k}^{(s)}=0$.

Придерживаясь вариационного подхода [19], введем анзац (22) в лагранжиан и выполним интегрирование по переменной $x$. Опуская детали вычислений, приведем ответ:

$$
\begin{gathered}
\mathcal{L}=\sum_{k=1}^{N} \mathcal{L}_{k}+\sum_{k=1}^{N} \sum_{n=k \pm 1} \tilde{\mathcal{L}}_{k n}, \\
\mathcal{L}_{k}=4 \nu_{k}\left(\frac{i}{2}\left(\left\langle\vec{n}_{k}, \vec{n}_{k, t}\right\rangle-\left\langle\vec{n}_{k, t}, \vec{n}_{k}\right\rangle\right)+2 \mu_{k} \frac{d \xi_{k}}{d t}-\frac{d \delta_{k}}{d t}-2 \mu_{k}^{2}+\frac{2 \nu_{k}^{2}}{3}\right), \\
\mathcal{L}_{k n}=16 \nu_{0}^{3} e^{-\Delta_{k n}}\left(R_{k n}+R_{k n}^{*}\right)+O\left(\epsilon^{3 / 2}\right), \\
R_{k n}=e^{i\left(\tilde{\delta}_{n}-\tilde{\delta}_{k}\right)}\left\langle\vec{n}_{k}, \vec{n}_{n}\right\rangle, \quad \tilde{\delta}_{k}=\delta_{k}-2 \mu_{0} \xi_{k}, \quad \Delta_{k n}=2 s_{k n} \nu_{0}\left(\xi_{k}-\xi_{n}\right),
\end{gathered}
$$

где коэффициенты $s_{k n}$ те же, что и в уравнениях (8).

Все необходимые для получения $\mathcal{L}_{k}$ интегралы могут быть вычислены явно (см. приложение). В результате мы имеем члены трех различных по величине порядков: 1) члены порядка $\left.\Delta_{k n}^{2} e^{-\Delta_{k n}} ; 2\right)$ члены порядка $\left.\Delta_{k n} e^{-\Delta_{k n}} ; 3\right)$ члены порядка $e^{-\Delta_{k n}}$. Более детальное рассмотрение показывает, что члены вида 1 и 2 умножаются на множители порядка $e^{-\Delta_{k n}}$ вследствие эволюционных уравнений для солитонных параметров. Окончательно, пренебрегая всеми членами порядка $\epsilon^{3 / 2}$ и выше, приходим к следующему набору динамических уравнений для солитонных параметров:

$$
\begin{gathered}
\frac{d \xi_{k}}{d t}=2 \mu_{k}, \\
\frac{d \delta_{k}}{d t}=2 \mu_{k}^{2}+2 \nu_{k}^{2}, \\
\frac{d \nu_{k}}{d t}=8 \nu_{0}^{3} \sum_{n} e^{-\Delta_{k n}} i\left(R_{k n}-R_{k n}^{*}\right), \\
\frac{d \mu_{k}}{d t}=-8 \nu_{0}^{3} \sum_{n} e^{-\Delta_{k n}}\left(R_{k n}+R_{k n}^{*}\right), \\
\frac{d \vec{n}_{k}}{d t}=4 \nu_{0}^{2} i \sum_{n=k \pm 1} e^{-\Delta_{k n}}\left[e^{i\left(\tilde{\delta}_{n}-\tilde{\delta}_{k}\right)} \vec{n}_{n}-R_{k n} \vec{n}_{k}+e^{i\left(\tilde{\delta}_{n}-\tilde{\delta}_{k}\right)} \vec{n}_{n}+R_{k n}^{*} \vec{n}_{k}\right]+C_{k} \vec{n}_{k},
\end{gathered}
$$

где константы $C_{k}$ фиксируются связью $\left\langle\vec{n}_{k}, \vec{n}_{k}\right\rangle=1$ для всех $t$.

Следующий шаг заключается в том, чтобы оценить порядок величины различных членов в лагранжиане $\mathcal{L}$ и отбросить те из них, которые имеют порядок $\epsilon^{3 / 2}$ и $\epsilon^{2}$. Напомним, что параметр $\epsilon$ характеризует величину перекрытия соседних солитонов (см. (3)).

Поэтому из уравнений $(27)$ и (28) имеем

$$
\frac{d\left(\mu_{k}+i \nu_{k}\right)}{d t}=4 \nu_{0}\left[\left\langle\vec{n}_{k}, \vec{n}_{k-1}\right\rangle e^{q_{k}-q_{k-1}}-\left\langle\vec{n}_{k+1}, \vec{n}_{k}\right\rangle e^{q_{k+1}-q_{k}}\right] .
$$

Кроме того, из уравнений (25) и (26) следует, что (см. [8])

$$
\frac{d q_{k}}{d t}=-4 \nu_{0}\left(\mu_{k}+i \nu_{k}\right)
$$

3 Теоретическая и математическая физика, т. 151, № 3, 2007 г. 
В результате мы доказали утверждение работы [20]:

$$
\frac{d^{2} q_{k}}{d t^{2}}=16 \nu_{0}^{2}\left[\left\langle\vec{n}_{k+1}, \vec{n}_{k}\right\rangle e^{q_{k+1}-q_{k}}-\left\langle\vec{n}_{k}, \vec{n}_{k-1}\right\rangle e^{q_{k}-q_{k-1}}\right] .
$$

В случае $n=2$ это уравнение совпадает с уравнением (9). Уравнение (32) в совокупности с системой уравнений для векторов поляризации (29) дает правильное обобщение модели КЦТ для многокомпонентной системы НУШ.

Уравнения для векторов поляризации являются нелинейными, поэтому система уравнений для $Q_{k}$ и $\vec{n}_{k}$ в целом неинтегрируема. Однако все члены в правых частях эволюционных уравнений для векторов поляризации $\vec{n}_{k}$ имеют порядок $\epsilon$. Это позволяет в главном приближении пренебречь эволюцией векторов $\vec{n}_{k}$ и аппроксимировать эти векторы их начальными значениями. В результате получим, что $N$ солитонные взаимодействия для векторного НУШ в адиабатическом приближении определяются КЦТ (см. раздел 3).

\section{6. ЗАКЛЮЧЕНИЕ}

Хорошо известно, что эффект двойного лучепреломления описывается двухкомпонентным обобщением НУШ:

$$
\begin{aligned}
& i u_{1 t}+\frac{1}{2} u_{1 x x}+\left(\left|u_{1}\right|^{2}+\beta\left|u_{2}\right|^{2}\right) u_{1}(x, t)=i r(x, t), \\
& i u_{2 t}+\frac{1}{2} u_{2 x x}+\left(\left|u_{2}\right|^{2}+\beta\left|u_{1}\right|^{2}\right) u_{2}(x, t)=i s(x, t),
\end{aligned}
$$

где параметр $\beta$ характеризует двойное лучепреломление, а функции $i r(x, t)$ и $i s(x, t)$ описывают дополнительные физические эффекты. Например, выбор $\operatorname{ir}(x, t)=$ $c u_{2}(x, t), i s(x, t)=c u_{1}(x, t)$ позволит учесть внутриканальное взаимодействие.

Известно, что данная система в случае Манакова $\beta=1, r=s=0$ (1) является интегрируемой. Однако в случае малых $r$ и $s$ и при $\beta$, близком к единице, можно рассматривать систему (33) как возмущенную систему Манакова. Применяя любой из описанных выше подходов, можно вывести возмущенный вариант КЦТ, который описывает $N$-солитонные взаимодействия в адиабатическом приближении.

Другой важной проблемой является анализ физических следствий, которые могут быть выведены из модели КЦТ. Эти задачи станут предметом последующих публикаций.

\section{ПРИЛОЖЕНИЕ}

Приведем здесь несколько типичных интегралов, которые возникают при вычислении лагранжиана:

$$
\begin{aligned}
K(a, \Delta) & \equiv \int_{-\infty}^{\infty} \frac{d z e^{i a z}}{2 \operatorname{ch} z \operatorname{ch}(z+\Delta)}=\frac{\pi\left(1-e^{-i a \Delta}\right)}{2 i \operatorname{sh} \Delta \operatorname{sh}(\pi a / 2)} \\
L(a, \Delta) & \equiv \int_{-\infty}^{\infty} \frac{d z e^{i a z} \operatorname{sh} z}{2 \operatorname{ch}^{2} z \operatorname{ch}(z+\Delta)}= \\
& =\frac{\pi i}{2 \operatorname{sh}^{2} \Delta \operatorname{sh}(\pi a / 2)}\left[\left(1-e^{-i a \Delta}\right) \operatorname{ch} \Delta-i a \operatorname{sh} \Delta\right]
\end{aligned}
$$




$$
\begin{aligned}
\tilde{L}(a, \Delta) & \equiv \int_{-\infty}^{\infty} \frac{d z e^{i a z} \operatorname{sh}(z+\Delta)}{2 \operatorname{ch} z \operatorname{ch}^{2}(z+\Delta)}=e^{-i a \Delta} L(a,-\Delta), \\
M(a, \Delta) & \equiv \int_{-\infty}^{\infty} \frac{d z e^{i a z} \operatorname{sh} z \operatorname{sh}(z+\Delta)}{2 \operatorname{ch}^{2} z \operatorname{ch}^{2}(z+\Delta)}= \\
& =\frac{\pi e^{-i a \Delta / 2}}{\operatorname{sh} \Delta \operatorname{sh}(\pi a / 2)}\left[a \cos \left(\frac{a \Delta}{2}\right) \frac{\operatorname{ch} \Delta}{\operatorname{sh} \Delta}-\sin \left(\frac{a \Delta}{2}\right)\left(1+\frac{2}{\operatorname{sh}^{2} \Delta}\right)\right] .
\end{aligned}
$$

В адиабатическом пределе $a \simeq 0$

$$
\begin{gathered}
K(0, \Delta)=\frac{\Delta}{\operatorname{sh} \Delta}, \\
L(0, \Delta) \equiv-\tilde{L}(0, \Delta)=\frac{1}{\operatorname{sh} \Delta}\left[1-\frac{\Delta \operatorname{ch} \Delta}{\operatorname{sh} \Delta}\right], \\
M(0, \Delta)=\frac{1}{\operatorname{sh}^{3} \Delta}\left[2 \operatorname{ch} \Delta \operatorname{sh} \Delta-\Delta \operatorname{sh}^{2} \Delta-2 \Delta\right] .
\end{gathered}
$$

В некоторых случаях необходимы также производные по $a$ при $a=0$ :

$$
\begin{aligned}
K_{1}(\Delta) & =\lim _{a \rightarrow 0} \frac{1}{i} \frac{d K(a, \Delta)}{d a} \equiv \int_{-\infty}^{\infty} \frac{d z z}{2 \operatorname{ch} z \operatorname{ch}(z+\Delta)}=-\frac{\Delta^{2}}{2 \operatorname{sh} \Delta}, \\
L_{1}(\Delta) & =\lim _{a \rightarrow 0} \frac{1}{i} \frac{d L(a, \Delta)}{d a} \equiv \int_{-\infty}^{\infty} \frac{d z z \operatorname{sh} z}{2 \operatorname{ch}^{2} z \operatorname{ch}(z+\Delta)}=\frac{\Delta^{2} \operatorname{ch} \Delta}{2 \operatorname{sh}^{2} \Delta}, \\
\tilde{L}_{1}(\Delta) & =\lim _{a \rightarrow 0} \frac{1}{i} \frac{d \tilde{L}(a, \Delta)}{d a} \equiv \int_{-\infty}^{\infty} \frac{d z z \operatorname{sh}(z+\Delta)}{2 \operatorname{ch} z \operatorname{ch}^{2}(z+\Delta)}=\frac{\Delta^{2} \operatorname{ch} \Delta}{2 \operatorname{sh}^{2} \Delta}, \\
M_{1}(\Delta) & =\lim _{a \rightarrow 0} \frac{1}{i} \frac{d M(a, \Delta)}{d a} \equiv \int_{-\infty}^{\infty} \frac{d z z \operatorname{sh} z \operatorname{sh}(z+\Delta)}{2 \operatorname{ch}^{2} z \operatorname{ch}^{2}(z+\Delta)}= \\
& =\frac{\Delta\left[-2 \operatorname{ch} \Delta \operatorname{sh}^{2} \Delta+\Delta \operatorname{sh}^{2} \Delta+2 \Delta\right]}{2 \operatorname{sh}^{3} \Delta}
\end{aligned}
$$

При вычислении $\mathcal{L}_{k n}$ нужно, разумеется, заменить $\Delta$ на $\Delta_{k n}$ и удержать лишь главные члены.

Благодарности. В.С. Герджиков выражает благодарность Bulgarian Science Foundation за частичную финансовую поддержку работы (грант № F-1410).

\section{Список литературы}

[1] Yu. S. Kivshar, G. P. Agrawal, Optical Solitons: From Fibers to Photonic Crystals, Academic Press, San Diego, 2003.

[2] C. Desem, P.L. Chu, "Soliton-soliton interactions", Optical Solitons - Theory and Experiment, ed. J.R. Taylor, Cambridge Univ. Press, Cambridge, 1992, 127; I. M. Uzunov, V. D. Stoev, T. I. Tzoleva, Optics Lett., 17 (1992), 1417.

[3] M. Suzuki, H. Toga, N. Edagawa, H. Tanaka, S. Yamamote, S. Akiba, Electron. Lett., 30 (1994), 1083.

[4] V.I. Karpman, V. V. Solov'ev, Phys. D, 3 (1981), 487.

[5] I. M. Uzunov, V.S. Gerdjikov, M. Gölles, F. Lederer, Opt. Commun., 125 (1996), 237. 
[6] V.S. Gerdjikov, D. J. Kaup, I. M. Uzunov, E. G. Evstatiev, Phys. Rev. Lett., 77 (1996), 3943; V.S. Gerdjikov, I. M. Uzunov, E. G. Evstatiev, G.L. Diankov, Phys. Rev. E, 55 (1997), 6039; V.S. Gerdjikov, I. M. Uzunov, Phys. D, 152-153 (2001), 355.

[7] V.S. Gerdjikov, E. V. Doktorov, J. Yang, Phys. Rev. E, 64 (2001), 056617.

[8] V.S. Shchesnovich, Phys. Rev. E, 65 (2002), 046614.

[9] E. V. Doktorov, N. P. Matsuka, V. M. Rothos, Phys. Rev. E, 69 (2004), 056607.

[10] J. M. Arnold, J. Opt. Soc. Amer. A, 15 (1998), 1450; Phys. Rev. E, 60 (1999), 979.

[11] С. В. Манаков, ЖЭТФ, 38 (1974), 248.

[12] В.Е. Захаров, С. В. Манаков, С.П. Новиков, Л.П. Питаевский, Теория солитонов. Метод обратной задачи, Наука, М., 1980.

[13] M. J. Ablowitz, B. Prinari, A. D. Trubatch, Discrete and Continuous Nonlinear Schrödinger Systems, London Math. Soc. Lect. Notes Ser., 302, Cambridge Univ. Press, Cambridge, 2004.

[14] J. Yang, Phys. Rev. E, 65 (2002), 036606.

[15] M. Midrio, S. Wabnitz, P. Franco, Phys. Rev. E, 54 (1996), 5743; V.S. Shchesnovich, E. V. Doktorov, Phys. Rev. E, 55 (1997), 7626; T. I. Lakoba, D. J. Kaup, Phys. Rev. E, 56 (1997), 6147; S. M. Baker, J. N. Elgin, J. Gibbons, Phys. Rev. E, 62 (1999), 4325.

[16] M. Toda, Theory of Nonlinear Lattices, Springer, Berlin, 1989; J. Moser, "Finitely many mass points on the line under the influence of an exponential potential - an integrable system", Dynamical Systems, Theory and Applications (Rencontres, BattelleRes. Inst., Seattle, Wash., 1974), Lect. Notes Phys., 38, ed. J. Moser, Springer, Berlin, 1975, 467; Adv. Math., 16 (1975), 197.

[17] V.S. Gerdjikov, E. G. Evstatiev, R. I. Ivanov, J. Phys. A, 31 (1998), 8221; 33 (2000), 975; solv-int/9909020.

[18] D. J. Kaup, V.S. Gerdjikov, E. G. Evstatiev, G. L. Diankov, I. M. Uzunov, Criterion and regions of stability for quasi-equidistant soliton trains, Preprint INRNE-TH-97-4, Institute for Nuclear Research and Nuclear Energy, Sofia, 1997; solv-int/9708004; V. S. Gerdjikov, E. G. Evstatiev, D. J. Kaup, G. L. Diankov, I. M. Uzunov, Phys. Lett. A, 241 (1998), 323.

[19] D. Anderson, Phys. Rev. A, 27 (1983), 3135; D. Anderson, M. Lisak, T. Reichel, Phys. Rev. A, 38 (1988), 1618; B. A. Malomed, Progr. Opt., 43 (2002), 69.

[20] V.S. Gerdjikov, " $N$-soliton interactions, the complex Toda chain and stability of NLS soliton trains", Proc. of the XVI-th Int. Symp. on Electromagnetic Theory, v. 1 (Aristotle Univ. of Thessaloniki, Greece, 1998), ed. E. Kriezis, 1998, 307. 